\title{
In vivo Direct Reprogramming of Fibroblasts into Cardiomyocyte-Like Cells Through Inhibition of HDACs and TGF- $\beta$ Pathway
}

\author{
Hua $\mathrm{Li}^{1}$, Junbo $\mathrm{Ge}^{2,3}$, and Gang Pei ${ }^{14^{*}}$
}

${ }^{1}$ State Key Laboratory of Cell Biology, Institute of Biochemistry and Cell Biology, Shanghai Institutes for Biological Sciences, Chinese Academy of Sciences, Shanghai 200031, China

${ }^{2}$ Shanghai Institute of Cardiovascular Diseases, Zhongshan Hospital, Fudan University, Shanghai 200032, China

3Institutes of Biomedical Sciences, Fudan University, Shanghai 200032, China

${ }^{4}$ School of Life Science and Technology, Tongji University, Shanghai 200092, China

\section{Abstract}

Background: Substantial progress has been recently achieved that direct lineage reprogramming induced by small molecule compounds was possible either in vitro or in vivo, which provided a promising cellular strategy for regenerative therapy.

Method: A combination of an inhibitor of HDACs, valproic acid (V) and an inhibitor of TGF- $\beta$ pathway, tranilast (T) were applied to identify the conversation of fibroblasts into induced cardiomyocyte-like cells (iCMs) in situ in mice with myocardial infarction.

Result: We found that the combination of two small molecules, V\&T could reprogram cardiac fibroblasts into iCMs in vivo, which were co-labeled with vimentin and a-actin 4 weeks after myocardial infarction; while, the phenomenon was found neither in mice with non-myocardial infarction nor in mice with myocardial infarction induced only by physiological saline. And furthermore, these iCMs resembled mouse native cardiomyocytes regarding their specific molecular phenotypes: a-MHC, c-TnT, connexin-43. However, the early marker of progenitor cells prior to cardiac differentiation, Mesp1, wasn't detected in the infarcted and border zone.

Conclusion: HDACs and TGF- $\beta$ inhibitors jointly could achieve direct cardiac reprogramming from cardiac fibroblasts in vivo, without establishing a pluripotent state and thus, provide a new important therapeutic application for cardiac regeneration.

Keywords: HDACs; Valproic acid; TGF- $\beta$; Tranilast; Induced cardiomyocyte-like cells; Direct reprogramming

\section{Introduction}

Reprogramming of resident support cells into alternative cells holds great promise as a novel therapy for the treatment of some diseases caused by irreversible loss of function within damaged organs. Direct reprogramming of fibroblasts into Induced Cardiomyocyte-like Cells (iCMs) provides a valuable cellular source for use in population with Myocardial Infarction (MI) or heart failure. Although some controversial issues about the effectiveness of cardiac reprogramming need further research [1], great progress in the target organ has been reported by several teams $[2,3]$. The main objective of present research was to describe the first use of a combination of an inhibitor of HDACs, Valproic acid (V) and an inhibitor of TGF- $\beta$ pathway, Tranilast (T) as a potential therapeutic tool for the conversion of adult mouse cardiac reprogramming.

\section{Methods}

Normal C57BL/6J mice (20-25 g, male, 8 weeks; Slac Laboratory, Shanghai, China) were housed under standard conditions in an animal room with a $12 / 12 \mathrm{~h}$ day/night cycle with free access to water and food. V\&T were obtained from Sigma-Aldrich. And V\&T were diluted in normal $(0.9 \%)$ saline to the desired concentration immediately before administration. Surgery to induced myocardial infarction was performed in the mice as described previously [4]. The mice were anesthetized by inhalation of $1.0-2.0 \%$ isoflurane gas while being mechanically ventilated on a positive pressure ventilator. Left thoracotomy was performed at the fourth intercostal space, and the pericardium was stripped to expose the heart. The left descending coronary artery was identified and occluded with an 8-0 silk ligature that was placed around it. Subsequently, the chest cavity was closed, and the animal was placed in a cage on a heating pad. Sham-operated mice underwent the same surgical procedures except that the suture placed under the left anterior descending artery was not tied. Three groups were used: Group1, Sham+Saline: each mouse received $1 \mathrm{mg} / \mathrm{kg}$ normal (0.9\%) saline (i.p.) 5 minutes before surgery and 60 minutes after sham-operation; Group2, MI+Saline: $1 \mathrm{mg} / \mathrm{kg}$ normal (0.9\%) saline (i.p.) was administered 5 minutes before surgery and 60 minutes after MI-operation; and group3, MI+V\&T: mice received $300 \mathrm{mg} / \mathrm{kg} \mathrm{V}$ and $200 \mathrm{mg} / \mathrm{kg}$ T (i.p.) 5 minutes before surgery and 60 minutes after MIoperation. The protocol was approved by the Committee on the Ethics of Animal Experiments of Fudan University. Each selected cardiac fragment 4 weeks after operation was processed routinely with primary antibody probing of a-actin (Abcam, ab5694), vimentin (Abcam, ab92547, ab8978), a-MHC (Abcam, ab50967), c-TnT (Abcam, ab8295), connexin-43 (Abcam, ab11370), and Mesp1 (Abcam, ab173011) at $4^{\circ} \mathrm{C}$ overnight, respectively. Appropriate fluorophore-conjugated secondary antibodies were used to visualize the expression in immunofluorescent

*Corresponding author: Gang Pei, State Key Laboratory of Cell Biology, Institute of Biochemistry and Cell Biology, Shanghai Institutes for Biological Sciences, Chinese Academy of Sciences, Shanghai 200031, China, Tel: +86-21-54921371; E-mail: gpei@sibs.ac.cn

Received January 10, 2017; Accepted January 20, 2017; Published January 27 2017

Citation: Hua Li, Junbo Ge, Gang Pei (2017) In vivo Direct Reprogramming of Fibroblasts into Cardiomyocyte-Like Cells Through Inhibition of HDACs and TGF- $\beta$ Pathway. J Stem Cell Res Ther 7: 373. doi: 10.4172/2157-7633.1000373

Copyright: (c) $2017 \mathrm{Hua} \mathrm{Li}$, et al. This is an open-access article distributed unde the terms of the Creative Commons Attribution License, which permits unrestricted use, distribution, and reproduction in any medium, provided the original author and source are credited. 
Citation: Hua Li, Junbo Ge, Gang Pei (2017) In vivo Direct Reprogramming of Fibroblasts into Cardiomyocyte-Like Cells Through Inhibition of HDACs and TGF- $\beta$ Pathway. J Stem Cell Res Ther 7: 373. doi: 10.4172/2157-7633.1000373

images that were captured by a TCS SP2 confocal microscope (Leica Microsystems, Wetzlar, Germany).

\section{Results}

Immunofluorescent staining for cardiomyocyte marker revealed expression of $\alpha$-actin, with sarcomere structure obviously visible; and staining for vimentin, a marker labeling fibroblasts, was scattered around cardiomyocytes (Figure 1A). High magnification view of white boxed area in Figure 1A showed no dual-labeled cardiomyocyte-like cells originated from fibroblasts in mice with non-MI (Figure 1B). No coexpression of $\alpha$-actin and vimentin was detected in the distant or border zone of mice with MI; however, physiological structure of heart was replaced with representatively pathological: cardiomyocytes disorder and fibroblasts irregularly proliferated (Figure 2). While, in mice treated with V\&T 4 weeks post-MI, iCMs originated from fibroblasts were identified that this population maintained vimentin expression and was stained a-actin positive, with sarcomeric structures (Figures 3A-3C). Furthermore, besides a-actin, immunofluorescent staining for cardiac markers, including a-MHC, c-TnT and connexin-43, also colabelled with vimentin in same sections from the border zone of cardiac infarction (Figures 4A-4C). Nevertheless, only transiently responsible for cardiac progenitors in the early stage, Mesp1, was not detected in the same region, suggesting that the V\&T-induced reprogramming process did not involve a pluripotent stem cell intermediate (Figure 4D).

\section{Discussion}

As inhibition of HDAC activity prevents global deacetylation and methylation at $\mathrm{H} 3 \mathrm{~K} 9$ residue at the promoters [5], treatment of cardiac fibroblasts by using inhibitions of HDACs and TGF- $\beta$ pathway might lead to gain of global histone acetylation and lose of $\mathrm{H} 3 \mathrm{~K} 9 \mathrm{me}$ in the cells. In addition, since the levels of histone acetylation and H3K9 methylation are positively and negatively associated with accessible chromatin state [6,7], cells treated with HDACs and TGF- $\beta$ pathway inhibitions are very likely to display a more open chromatin state, which is strongly relative to the pluripotent state [8]. Interestingly, as histone $\mathrm{H} 3 \mathrm{~K} 9$ methyltransferases recently have been shown to be able to interact with DNA methyltransferases and maintain DNA methylation at specific loci [9], blockade the chromatin binding of H3K9 methyltransferases might reduce the recruitment of DNA methyltransferases and further result loss of DNA methylation loci specifically or globally. Therefore, investigation the levels of histone acetylation, $\mathrm{H} 3 \mathrm{~K} 9$ methylation, as long as DNA methylation in the

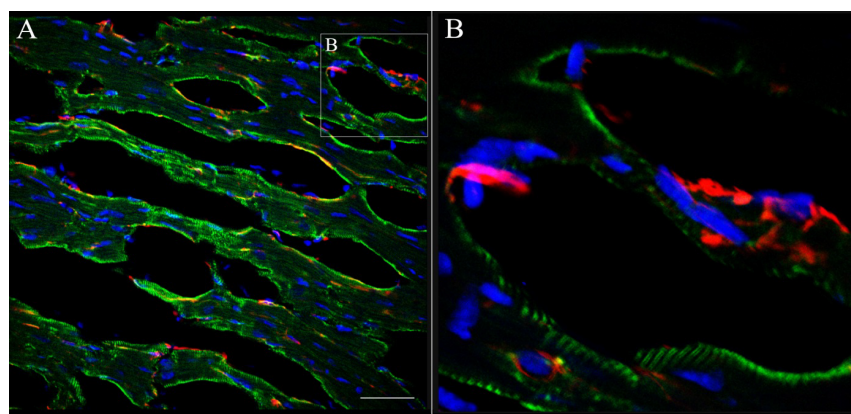

Figure 1: Dual-labeled cardiomyocyte-like cells originated from fibroblasts in mice with non-myocardial infarction. A: GFP conjugated second antibody against the primary antibody a-actin, and RFP conjugated second antibody against the primary antibody vimentin, as well as DAPI, in the cardiomyocytes and fibroblasts, respectively. B: High magnification view of selectively boxed area in Figure1.A showed no both a-actin GFP expression and positive sarcomeric organization in fibroblasts (RFP). Scale bar represents $20 \mu \mathrm{m}$.

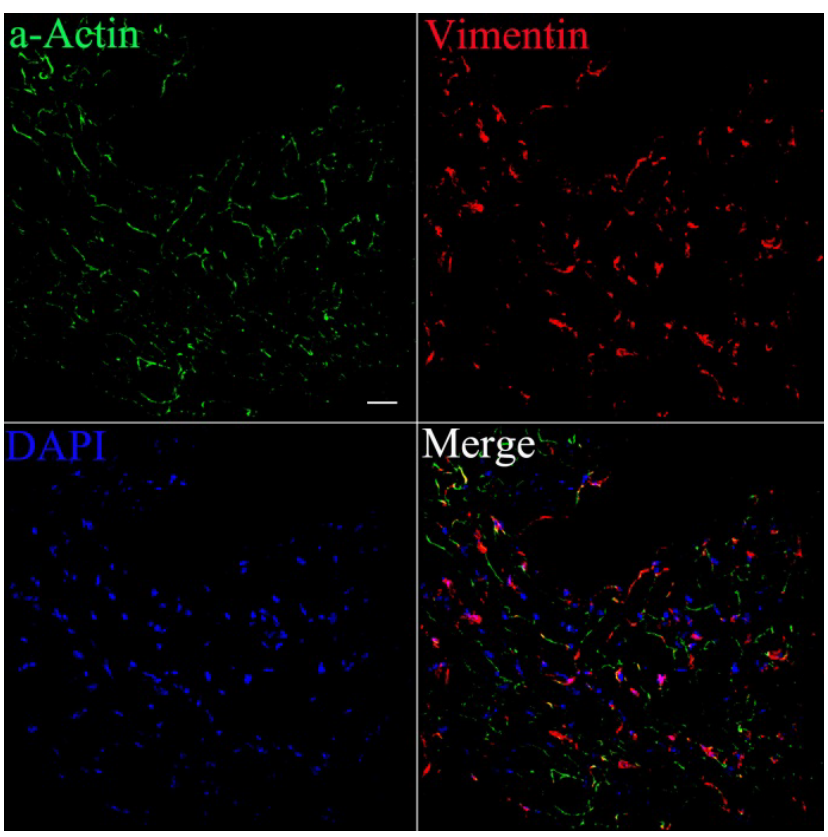

Figure 2: Dual-labeled cardiomyocyte-like cells originated from fibroblasts in mice with myocardial infarction. GFP conjugated second antibody against the primary antibody a-actin in the cardiomyocytes and RFP conjugated second antibody against the primary antibody vimentin in the fibroblasts in mice with myocardial infarction were shown. However, tissue structure of myocardial infarcted zone was not physiological, instead of representatively pathological: cardiomyocytes disorder and fibroblasts irregularly proliferated. Merge view was from distant or border zones where endogenous cardiomyocytes were labelled by a-actin, but were never co-localized with vimentin. Scale bar represents $20 \mu \mathrm{m}$

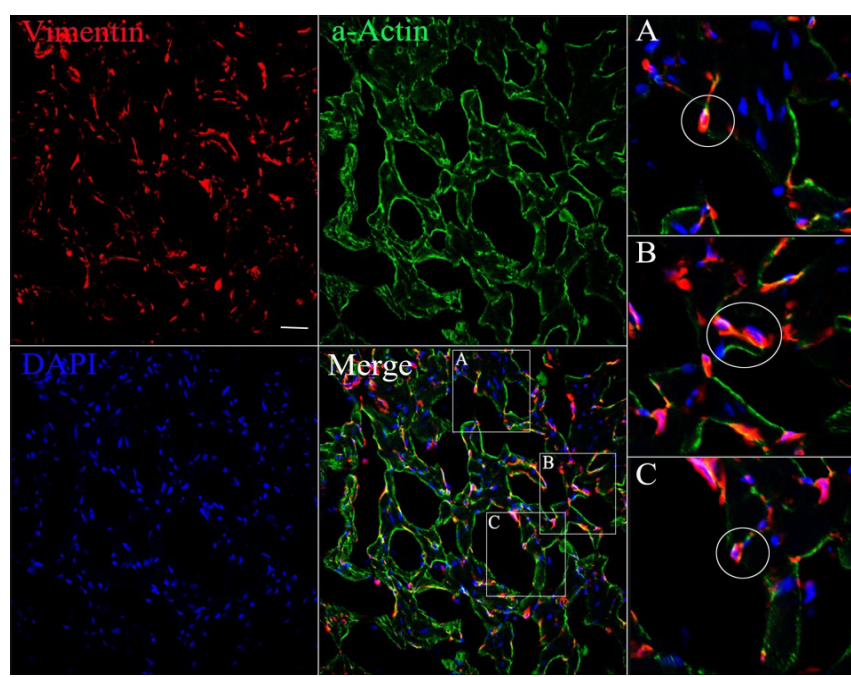

Figure 3: Dual-labeled VT-induced cardiomyocyte-like cells originated from fibroblasts in mice with myocardial infarction. RFP conjugated second antibody against the primary antibody vimentin and GFP conjugated second antibody against the primary antibody a-actin in fibroblast-derived cardiomyocyte-like cells induced by VT 4 weeks after myocardial infarction. A, B\&C: White boxes in the merged picture indicated induced cardiomyocyte-like cells maintained vimentin expression, were co-localized with a-actin and had sarcomeric structures. V: Valproic acid, T: Tranilast. Scale bar represents $20 \mu \mathrm{m}$. 
Citation: Hua Li, Junbo Ge, Gang Pei (2017) In vivo Direct Reprogramming of Fibroblasts into Cardiomyocyte-Like Cells Through Inhibition of HDACs and TGF- $\beta$ Pathway. J Stem Cell Res Ther 7: 373. doi: 10.4172/2157-7633.1000373
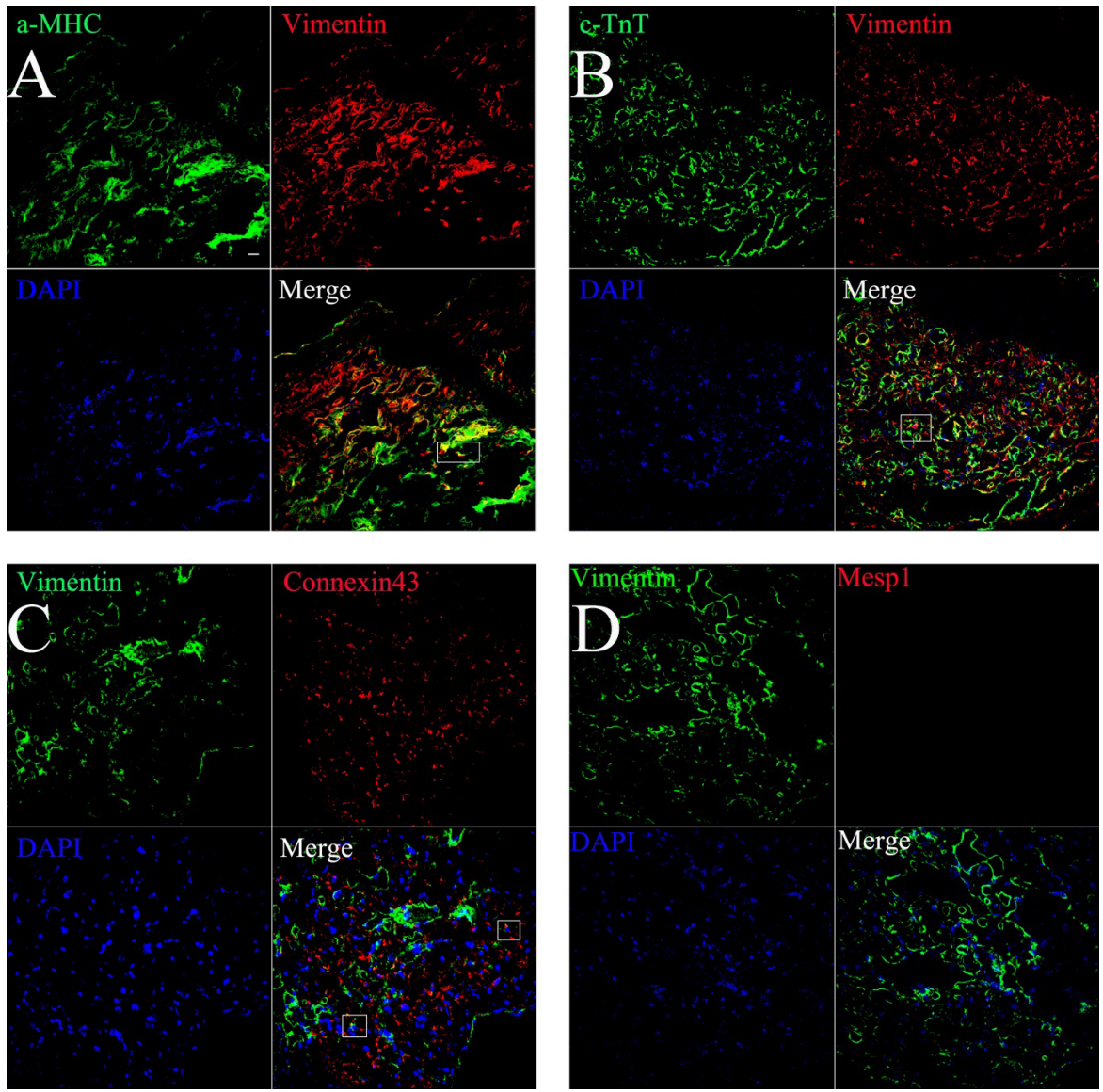

Figure 4: Dual-labeled VT-induced cardiomyocyte-like cells with vimentin and a-MHC, c-TnT, Connexin-43, Mesp1 in infarcted mice. A, B\&C: Immunofluorescent staining for cardiac markers, besides a-actin, including a-MHC, c-TnT and connexin-43, also co-labelled with vimentin and DAPI, in frozen sections from the border zone of cardiac infarction. The white boxes inset in the merged figures displayed representative reprogrammed cardiomyocyte-like cells. D: Mesp1, transiently responsible for cardiac progenitors, was not found co-expression with vimentin in fibroblasts induced by VT. V: Valproic acid, T: Tranilast. Scale bar represents $20 \mu \mathrm{m}$.

cardiac fibroblasts treated with HDACs and TGF- $\beta$ pathway inhibitions might be a new direction to deeper understand the mechanisms that underlying the reprogramming of these cells.

The present study focused on an optimized and convenient method for the conversation of cardiac fibroblasts into iCMs under hypoxia, and determined whether a combination of an inhibitor of HDACs, valproic acid and an inhibitor of TGF- $\beta$ pathway, tranilast was effective at direct in situ reprogramming of cardiac fibroblasts. Our finding demonstrated for the first time that iCMs remained vimentin, a marker of cardiac fibroblasts, and concurrently expressed markers of cardiomyocytes, a-actin, a-MHC, c-TnT and connexin-43, which was consistent with previous research of reprogramming in fibroblasts [10,11]. Furthermore, Mesp1, expressing in the early stage of cardiac progenitors, was not detected, suggesting that the chemical molecular induced reprogramming process did not involve a pluripotent state. Besides, valporic acid is an anti-epileptic drug widely used to treat partial and generalized seizures, especially in children [12]. And tranilast, an anti-allergic drug, that has been used for more than 30 years in clinical practice [13]. These evidences gave us no doubt about the safety of in vivo reprogramming strategies. Based on this evidence, a novel therapeutic action for cardiac diseases via loss of function may be applied into future clinic medicine though further and detailed research.

\section{Acknowledgements}

This work was supported by the National Nature Science Foundation of China (No.81500229) and China Postdoctoral Science Foundation (No.2015T80464).

\section{Conflicts of Interest}

The authors confirm that there are no conflicts of interest.

\section{References}

1. Chien KR, Yi BA, Xu H, Mummery CL (2012) Cardiomyocyte reprogramming and the new age of cellular alchemy. J Mol Cell Cardiol 53: 311-313. [PubMed]

2. Jayawardena TM, Egemnazarov B, Finch EA, Zhang L, Payne JA, et al. (2012)MicroRNA-mediated in vitro and in vivo direct reprogramming of cardiac fibroblasts to cardiomyocytes. Circ Res 110: 1465-1473. [PubMed]

3. Song K, Nam YJ, Luo X, Qi X, Tan W, et al. (2012) Heart repair by reprogramming non-myocytes with cardiac transcription factors. Nature 485: 599-604. [PubMed]

4. Zhao G, Wang S, Wang Z, Sun A, Yang X, et al. (2013) CXCR6 deficiency ameliorated myocardial ischemia/reperfusion injury by inhibiting infiltration of monocytes and IFN-gamma-dependent autophagy. Int J Cardiol 168: 853862. [PubMed]

5. Fang S, Miao J, Xiang L, Ponugoti B, Treuter E, et al. (2007) Coordinated recruitment of histone methyltransferase G9a and other chromatin-modifying enzymes in SHP-mediated regulation of hepatic bile acid metabolism. Mol Cell Biol 27: 1407-1424. [PubMed] 
Citation: Hua Li, Junbo Ge, Gang Pei (2017) In vivo Direct Reprogramming of Fibroblasts into Cardiomyocyte-Like Cells Through Inhibition of HDACs and TGF- $\beta$ Pathway. J Stem Cell Res Ther 7: 373. doi: 10.4172/2157-7633.1000373

6. Görisch SM, Wachsmuth M, Tóth KF, Lichter P, Rippe K (2005) Histone acetylation increases chromatin accessibility. J Cell Sci 118: 5825-5834. [PubMed]

7. Stewart MD, Li J, Wong J (2005) Relationship between histone H3 lysine 9 methylation, transcription repression, and heterochromatin protein 1 recruitment. Mol Cell Biol 25: 2525-2538. [PubMed]

8. Wu J, Huang B, Chen H, Yin Q, Liu Y, et al. (2016) The landscape of accessible chromatin in mammalian preimplantation embryos. Nature 534 652-657. [PubMed]

9. Zhang T, Termanis A, Özkan B, Bao XX, Culley J, et al. (2016) G9a/GLP Complex Maintains Imprinted DNA Methylation in Embryonic Stem Cells. Cell Reports 15: 77-85. [PubMed]
10. leda M, Fu JD, Delgado-Olguin P, Vedantham V, Hayashi Y, et al. (2010) Direct reprogramming of fibroblasts into functional cardiomyocytes by defined factors. Cell 142: 375-386. [PubMed]

11. Qian L, Huang $\mathrm{Y}$, Spencer $\mathrm{Cl}$, Foley A, Vedantham $\mathrm{V}$, et al. In vivo reprogramming of murine cardiac fibroblasts into induced cardiomyocytes. Nature 485: 593-598.

12. Shiari R, Bagherzade L, Alaei MR (2011) Fanconi Syndrome Associated with Valporic Acid: A Case Report. Iran Red Crescent Med J 13: 844-845. [PubMed]

13. Hasegawa S, Kitoh H, Ohkawara B, Mishima K, Matsushita M, et al. (2016) Tranilast stimulates endochondral ossification by upregulating SOX9 and RUNX2 promoters. Biochem Biophys Res Commun 470: 356-361. [PubMed] 